\title{
On simple Lie algebras of dimension seven over fields of characteristic 2
}

\author{
Alexandre N. Grichkov * \\ Departamento de Matemática, Instituto de Matemática e Estatística, \\ Universidade de São Paulo, S.P., Brasil

\section{Marinês Guerreiro ${ }^{\dagger}$} \\ Departamento de Matemática, Centro de Ciências Exatas e Tecnológicas, \\ Universidade Federal de Viçosa, M.G., Brasil
}

\section{Introduction}

The problem of classification of the simple Lie algebras over a field of characteristic $p>7$ was solved in the middle of the 90's by H. Strade, R. Block and R. L. Wilson (see [B], [BW1], [BW2], [SW], [S89.1], [S92], [S92.1], [Wi]). In the beginning of the 2000's, A. Premet and H. Strade proved the classification results for $p=5$ and 7 in a series of papers [PS1], [PS2], [PS3], but for $p=2$ and $p=3$ the problem is still open. Throughout this paper all algebras are defined over a fixed algebraically closed field $k$ of characteristic 2 containing the prime field $\mathbb{F}_{2}$. We start with some basic definitions and known facts.

Definition 1.1. A Lie algebra $L$ over $k$ is a Lie 2-algebra if there exists a map $L \rightarrow L, x \longmapsto x^{[2]}$, called 2-map, such that

$$
(x+\lambda y)^{[2]}=x^{[2]}+\lambda^{2} y^{[2]}+\lambda[x, y] \text {, for all } x, y \in L, \lambda \in k .
$$

It is well known fact that for every algebra $A$ over a field $k$ of characteristic 2 the corresponding Lie algebra $\operatorname{Der}_{k} A$ of $k$-derivations of $A$ has the natural structure of 2 -Lie algebra such that $d^{[2]}(a)=d^{2}(a)=d(d((a))$.

Definition 1.2. Let $L$ be a Lie algebra such that $Z(L)=0$, which is also called a centerless Lie algebra. The 2-closure of $L$ in $\operatorname{Der}_{k}(L)$,

* Supported by FAPEMIG, FAPESP, CNPq(Brazil) and RFFI, grant 07-01-00392A (Russian).

$\dagger$ Supported by FAPEMIG and FAPESP(Brazil) Processo N. 04/07774-2. 
denoted by $L_{2}$, is the smallest subalgebra of $\operatorname{Der}_{k}(L)$ containing $L$ and closed under the 2-map.

According to H. Strade [S89], the toral rank of $L$ is the maximal dimension $T(L)$ of the toral subalgebras of $L$. By definition, a toral subalgebra is an abelian subalgebra with a basis $\left\{t_{1}, \ldots, t_{n}\right\}$ such that $t_{i}^{[2]}=t_{i}, i=$ $1, \ldots, n$. The absolute toral rank $T R(L)$ of a centerless Lie algebra $L$ is $T\left(L_{2}\right)$ - toral rank of 2 -closure of $L$ defined above.

The first results for the classification problem in characteristic 2 are as follows.

Theorem 1.1 (S. Skryabin, [Sk]). Let $L$ be a simple finite dimensional Lie $k$-algebra over an algebraically closed field $k$ of characteristic 2 . Then $L$ has absolute toral rank greater or equal to 2 .

In the case of absolute toral rank 2, A. Grichkov and A. Premet announced the following result:

Theorem 1.2 (A. Premet, A. Grichkov [GP]). Let L be a simple Lie $k$-algebra of finite dimension with $k$ an algebraically closed field of characteristic 2. If the absolute toral rank of $L$ is 2 , then $L$ is classical of dimension 3, 8, 14 or 26.

The toral rank 3 is a much more difficult case and it is still open. In this work we begin the study of the simple Lie algebras of dimension seven and absolute toral rank 3 over an algebraically closed field $k$ of characteristic 2 .

In the literature up to this date there appeared only three types of the simple Lie 2-algebras of dimension 7 and absolute toral rank 3: the WittZassenhaus algebra $\overline{W(1 ; 3)}[\mathrm{Ju}]$, the Hamiltonian algebra $H_{2}$ [SF] (p. 144) (this algebra corresponds to a non-standard 2-form) and a family $L(\varepsilon)$, called the Kostrikin-Dzhumadil'daev algebras, that depends on one parameter $\varepsilon \in k[\mathrm{~K}]$. Here we calculate some features of these algebras such as their group of 2-automorphisms and their varieties of idempotent and nilpotent elements. We also present some Cartan decompositions for these algebras. The study of the algebras $W$ and $H_{2}$ is motivated by the following conjecture.

Conjecture 1.1. Let $L$ be a simple finite dimensional Lie algebra over an algebraically closed field of characteristic 2. If $\operatorname{dim} L>3$ then $L$ contains a subalgebra $\mathrm{W}$ or $\mathrm{H}_{2}$.

In this paper we prove that all simple Kostrikin-Dszumadil'daev 7dimensional Lie algebras are isomorphic to the Hamiltonian algebra $\mathrm{H}_{2}$. 
This is a reason why we sometimes use in this paper the notation $K$ instead of $\mathrm{H}_{2}$ for this algebra.

In a second paper we will prove that, for dimension 7 and absolute toral rank 3, a simple Lie 2-algebra is either isomorphic to a Witt-Zassenhaus or to a Hamiltonian algebra.

Definition 1.3. Let $L$ be a Lie 2-algebra. A k-linear map $\varphi: L \rightarrow L$ is a 2-automorphism of $L$ provided that $\varphi\left(x^{[2]}\right)=(\varphi(x))^{[2]}$ for all $x \in L$. Denote by $A_{u t}(L)$ the group of all 2-automorphisms of $L$.

Note that by definition of Lie 2-algebras, every 2-automorphism of a Lie 2 -algebra is an automorphism of $L$, but inverse is not true.

Throughout this paper we denote by $\bar{a}$ the element $a+1$, for $a \in k$, and $\langle M\rangle$ is the $k$-vector space spanned by the set $M$.

\section{The Witt-Zassenhaus algebra}

The simple Witt-Zassenhaus Lie algebra, denoted here by $W=\overline{W(1 ; 3)}$, can be constructed using different approaches as one can see in [Ju], [SF] or $[\mathrm{K}]$. Here we consider a basis $\left\{y_{i}:-1 \leq i \leq 5\right\}$ for $W$ and denote its 2 -closure in $\operatorname{Der}_{k}(W)$ by $W_{2}=\left\langle\eta, \kappa, \kappa^{[2]}, y_{i}:-1 \leq i \leq 5\right\rangle$. The Lie multiplication in $W_{2}$ is given by the table below. Note that the diagonal of this table exhibits the elements $x^{[2]}$, for each $x \in W_{2}$.

The 2-closure $W_{2}$ of the Witt-Zassenhaus algebra $W$

\begin{tabular}{|c||c|c|c|c|c|c|c|c|c|c|}
\hline \hline & $\eta$ & $\kappa$ & $\kappa^{[2]}$ & $y_{-1}$ & $y_{0}$ & $y_{1}$ & $y_{2}$ & $y_{3}$ & $y_{4}$ & $y_{5}$ \\
\hline \hline$\eta$ & 0 & $y_{4}$ & $y_{2}$ & $y_{5}$ & 0 & 0 & 0 & 0 & 0 & 0 \\
\hline$\kappa$ & $y_{4}$ & $\kappa^{[2]}$ & 0 & 0 & 0 & $y_{-1}$ & $y_{0}$ & $y_{1}$ & $y_{2}$ & $y_{3}$ \\
\hline$\kappa^{[2]}$ & $y_{2}$ & 0 & 0 & 0 & 0 & 0 & 0 & $y_{-1}$ & $y_{0}$ & $y_{1}$ \\
\hline$y_{-1}$ & $y_{5}$ & 0 & 0 & $\kappa$ & $y_{-1}$ & $y_{0}$ & $y_{1}$ & $y_{2}$ & $y_{3}$ & $y_{4}$ \\
\hline$y_{0}$ & 0 & 0 & 0 & $y_{-1}$ & $y_{0}$ & $y_{1}$ & 0 & $y_{3}$ & 0 & $y_{5}$ \\
\hline$y_{1}$ & 0 & $y_{-1}$ & 0 & $y_{0}$ & $y_{1}$ & $y_{2}$ & 0 & $y_{4}$ & $y_{5}$ & 0 \\
\hline$y_{2}$ & 0 & $y_{0}$ & 0 & $y_{1}$ & 0 & 0 & 0 & $y_{5}$ & 0 & 0 \\
\hline$y_{3}$ & 0 & $y_{1}$ & $y_{-1}$ & $y_{2}$ & $y_{3}$ & $y_{4}$ & $y_{5}$ & $\eta$ & 0 & 0 \\
\hline$y_{4}$ & 0 & $y_{2}$ & $y_{0}$ & $y_{3}$ & 0 & $y_{5}$ & 0 & 0 & 0 & 0 \\
\hline$y_{5}$ & 0 & $y_{3}$ & $y_{1}$ & $y_{4}$ & $y_{5}$ & 0 & 0 & 0 & 0 & 0 \\
\hline \hline
\end{tabular}

2.1. The group of 2-automorphisms $G_{1}=A u t_{k, 2}\left(W_{2}\right)$.

Proposition 2.1. The group $G_{1}$ of 2-automorphisms of $W_{2}$ is defined on the basis elements of $W_{2}$, for $\varphi=\varphi\left(\alpha_{-1}, \alpha_{1}, \alpha_{3}, \alpha_{4}, \alpha_{5}\right) \in G_{1}$ and 


$$
\begin{aligned}
\alpha_{-1} \neq 0, \text { by: } & \\
\varphi: y_{-1} & \longmapsto \alpha_{-1} y_{-1}+\alpha_{1} y_{1}+\alpha_{3} y_{3}+\alpha_{4} y_{4}+\alpha_{5} y_{5} \\
y_{0} & \longmapsto y_{0}+\alpha_{4} \alpha_{-1}^{-1} y_{5} \\
y_{1} & \longmapsto \alpha_{-1}^{-1} y_{1}+\alpha_{3} \alpha_{-1}^{-2} y_{5} \\
y_{2} & \longmapsto \alpha_{-1}^{-2} y_{2} \\
y_{3} & \longmapsto \alpha_{-1}^{-3} y_{3}+\alpha_{1} \alpha_{-1}^{-4} y_{5} \\
y_{4} & \longmapsto \alpha_{-1}^{-4} y_{4} \\
y_{5} & \longmapsto \alpha_{-1}^{-5} y_{5} \\
\eta & \longmapsto \alpha_{-1}^{-6} \eta \\
\kappa & \longmapsto \alpha_{-1}^{2} \kappa+\alpha_{3}^{2} \eta+\alpha_{-1} \alpha_{1} y_{0}+\left(\alpha_{1}^{2}+\alpha_{-1} \alpha_{3}\right) y_{2}+ \\
& \alpha_{-1} \alpha_{4} y_{3}+\left(\alpha_{1} \alpha_{3}+\alpha_{-1} \alpha_{5}\right) y_{4}+\alpha_{1} \alpha_{4} y_{5} \\
\kappa^{[2]} & \longmapsto \alpha_{-1}^{4} \kappa^{[2]}+\alpha_{-1}^{2} \alpha_{4}^{2} \eta+\alpha_{-1}^{3} \alpha_{3} y_{0}+\alpha_{-1}^{3} \alpha_{4} y_{1}+ \\
& \alpha_{-1}^{2}\left(\alpha_{1} \alpha_{3}+\alpha_{-1} \alpha_{5}\right) y_{2}+\alpha_{-1}^{2} \alpha_{3}^{2} y_{4}+\alpha_{-1}^{2} \alpha_{3} \alpha_{4} y_{5} .
\end{aligned}
$$

Note that $\operatorname{dim}_{k} G_{1}=5$ for every field $k$ of characteristic 2 .

Proof. It is not difficult to prove that, for all $0 \neq \alpha_{-1}, \alpha_{1}, \alpha_{2}, \alpha_{3}, \alpha_{4}, \alpha_{5} \in$ $k$, a map $\phi$ defined as in the proposition is a 2-automorphism of $W_{2}$. In order to prove that every 2-automorphism of $W_{2}$ is defined exactly like this, we first construct some $G_{1}$-invariant subspaces and subsets of $W_{2}$. Construct some $G_{1}$-invariant subspaces and subsets of $W_{2}$.

It is clear that all subsets defined below are $G_{1}$-invariant subsets. Note that $W=\left[W_{2}, W_{2}\right]$.

1. $V_{1}=\left\{x \in W: x^{[2]}=0\right\}=\operatorname{Span}_{k}\left\{y_{2}, y_{4}, y_{5}\right\}$,

2. $V_{2}=\left\{x \in W:\left[x, V_{1}\right] \subseteq V_{1}\right\}=\operatorname{Span}_{k}\left\{y_{0}, y_{1}, y_{2}, y_{3}, y_{4}, y_{5}\right\}$,

3. $V_{3}=\left[V_{2}, V_{2}\right]=\operatorname{Span}_{k}\left\{y_{1}, y_{3}, y_{4}, y_{5}\right\}$,

4. $V_{4}=\left[V_{3}, V_{3}\right]=\operatorname{Span}_{k}\left\{y_{4}, y_{5}\right\}$,

5. $V_{5}=\left\{x \in V_{3}:\left[x, V_{3}\right]=0\right\}=k y_{5}$,

6. $V_{6}=\left\{x \in V_{1}: \operatorname{dim}\left[x, W_{2}\right]=3\right\}=k y_{2}$.

Let $\psi$ be an arbitrary 2-automorphism of $W_{2}$. Since $V_{5}$ is $G_{1}$-invariant, we may suppose that $y_{5}^{\psi}=y_{5}, y_{-1}^{\psi}=\sum_{i=-1}^{5} r_{i} y_{i}$. By $\left[y_{-1}, y_{i}\right]=y_{i-1}$, $i=0, \ldots, 5$, we have

$y_{4}^{\psi}=r_{-1} y_{4}, y_{3}^{\psi}=r_{-1}^{2} y_{3}+r_{-1} r_{1} y_{5}, y_{2}^{\psi}=r_{-1}^{3} y_{2}+r_{-1}^{2} r_{0} y_{3}+r_{-1}\left(r_{0} r_{1}+r_{2} r_{-1}\right) y_{5}$.

Since $r_{-1} \neq 0$ and $V_{6}$ is $G_{1}$-invariant, $r_{0}=r_{2}=0$. Using some 2automorphism $\phi\left(\alpha_{-1}, \alpha_{1}, \alpha_{3}, \alpha_{4}, \alpha_{5}\right)$ we may suppose that $r_{0}=r_{1}=r_{2}=$ 
$r_{3}=r_{4}=r_{5}=0$. Hence,

$$
\begin{aligned}
y_{-1}^{\psi}=r_{-1} y_{-1}, & y_{4}^{\psi}=r_{-1} y_{4}, \quad y_{3}^{\psi}=r_{-1}^{2} y_{3}, \\
y_{2}^{\psi}=r_{-1}^{3} y_{2}, & y_{1}^{\psi}=r_{-1}^{4} y_{1}, \quad y_{0}^{\psi}=r_{-1}^{5} y_{0} .
\end{aligned}
$$

By $\left[y_{0}, y_{5}\right]=y_{5}$, we get $r_{-1}^{5}=1$. Then $\psi=\phi\left(r_{-1}, 0,0,0,0\right)$.

At last, $\eta^{\psi}=\left(y_{3}^{\psi}\right)^{[2]}, \kappa^{\psi}=\left(y_{-1}^{\psi}\right)^{[2]}$, since $\psi$ is an 2-automorphism.

2.2. Idempotent and Nilpotent Elements of $W_{2}$. The sets of nilpotent and idempotent elements of a Lie algebra are quite important features of the algebra structure as they allow us to construct different subalgebras and study the relations among them. In fact a method based on a study of the orbits of toral elements with respect to the automorphism group of the algebra and on an investigation of the centralizer of a toral element was already used in several papers describing the structure of tori and Cartan subalgebras of a Lie $p$-algebra, for a prime $p$, see [S92], [BW2] [R], [W].

Proposition 2.2. For the Lie 2 -algebra $W_{2}$, the variety of idempotent elements is given by $I(W)=\bigcup_{\delta=1}^{3} I_{W}^{\delta}$, where

$$
\begin{aligned}
I_{W}^{1}= & \left\{a^{4} \kappa^{[2]}+a^{2} \kappa+b^{2} \eta+a y_{-1}+c y_{0}+(\bar{c}+b) y_{1}+\left(\bar{c}^{2}+b+d\right) y_{2}+\right. \\
& \left.b y_{3}+d y_{4}+(\bar{c} b+d) y_{5}: a \in k^{*}, b, c, d \in k\right\}, \\
I_{W}^{2}= & \left\{a^{2} \eta+y_{0}+b y_{1}+b^{2} y_{2}+a y_{3}+a b y_{4}+c y_{5}: a \in k^{*}, b, c \in k\right\}, \\
I_{W}^{3}= & \left\{y_{0}+a y_{1}+a^{2} y_{2}+b y_{5}: a, b \in k\right\} .
\end{aligned}
$$

Moreover, $I_{W}^{1}=\left\{\kappa^{[2]}+\kappa+y_{-1}+y_{1}+y_{2}\right\}^{G_{1}}$; that is, all elements of $I_{W}^{1}$ belong to the same orbit under the $G_{1}$-action.

$I_{W}^{2}=\cup_{b \in k / \mathbf{Z}_{3}}\left\{\eta+y_{0}+b y_{1}+b^{2} y_{2}+y_{3}+b y_{4}\right\}^{G_{1}}$, where $\mathbf{Z}_{3}=\left\{1, \delta, \delta^{2}=\right.$ $1+\delta\}$.

$$
I_{W}^{3}=y_{0}^{G_{1}} \cup\left\{y_{0}+y_{1}+y_{2}\right\}^{G_{1}} .
$$

Proof. Let $t^{[2]}=t=b_{1} \kappa^{[2]}+b_{2} \kappa+b_{3} \eta+a y_{-1}+a_{0} y_{0}+a_{1} y_{1}+a_{2} y_{2}+$ $a_{3} y_{3}+a_{4} y_{4}+a_{5} y_{5}$. Comparing the coefficients at $k^{[2]}, \ldots, y_{5}$, by Table I we get:

$$
\begin{gathered}
b_{1}=b_{2}^{2}, b_{2}=a^{2}, b_{3}=a_{3}^{2}, \\
a=a^{4} a_{3}+b_{2} a_{1}+a a_{0}, \\
a_{0}=a_{0}^{2}+b_{1} a_{4}+a_{2} b_{2}+a a_{1}, \\
a_{1}=b_{1} a_{5}+b_{2} a_{3}+a a_{2}+a_{0} a_{1}, \\
a_{2}=b_{1} b_{3}+b_{2} a_{4}+a a_{3}+a_{1}^{2}, \\
a_{3}=b_{2} a_{5}+a a_{4}+a_{0} a_{3},
\end{gathered}
$$




$$
\begin{gathered}
a_{4}=b_{2} b_{3}+a a_{5}+a_{3} a_{1}, \\
a_{5}=a b_{3}+a_{1} a_{4}+a_{2} a_{3}+a_{0} a_{5},
\end{gathered}
$$

Note that $0 \neq t$ is an idempotent if and only if we have all equalities (1)-(8). By (1), we have $b_{1}=a^{4}$. Suppose that $a \neq 0$. Using (2) we get

$$
a_{0}=1+a a_{1}+a^{3} a_{3} \text {. }
$$

By (5) we get

By (7) we have

$$
a_{2}=a^{4} a_{3}^{2}+a^{2} a_{4}+a_{1}^{2}+a a_{3}
$$

$$
a_{4}=a a_{5}+a_{3} a_{1}+a^{2} a_{3}^{2}, a_{2}=a^{3} a_{5}+a^{2} a_{3} a_{1}+a_{1}^{2}+a a_{3} ;
$$

then $t=a^{4} \kappa^{[2]}+a^{2}+\kappa+a_{3}^{2} \eta+a y_{-1}+\left(1+a a_{1}+a^{3} a_{3}\right) y_{0}+a_{1} y_{1}+\left(a^{3} a_{5}+\right.$ $\left.a^{2} a_{3} a_{1}+a_{1}^{2}+a a_{3}\right) y_{2}+a_{3} y_{3}+\left(a a_{5}+a_{3} a_{1}+a^{2} a_{3}^{2}\right) y_{4}+a_{5} y_{5}$ is an idempotent.

In the case $a=0$ the calculations are analogous but more easy.

All statements about the conjugation of idempotents are easy to prove. For example, consider the set $I_{W}^{2}$. If $b=0$ then $t=a^{2} \eta+y_{0}+a y_{3}+c y_{5}=$ $\left(\eta+y_{0}+y_{3}\right)^{\phi}$, where $\phi=\phi(x, y, 0,0,0), x^{3}=1 / a, y=x c / a$. Suppose that $b \neq 0$. In this case $t=a^{2} \eta+y_{0}+b y_{1}+b^{2} y_{2}+a y_{3}+a b y_{4}+c y_{5}$ is conjugated with $t\left(b_{1}\right)=\eta+y_{0}+b_{1} y_{1}+b_{1}^{2} y_{2}+y_{3}+b_{1} y_{4}$. Suppose that $t\left(b_{1}\right)$ is conjugated with $t\left(b_{2}\right)=\eta+y_{0}+b_{2} y_{1}+b_{2}^{2} y_{2}+y_{3}+b_{2} y_{4}$, then $t\left(b_{1}\right)^{\phi}=t\left(b_{2}\right), \phi=\phi(x, y, z, p, q)$. Hence, $x^{3}=1$ and $b_{1} x=b_{2}$.

Proposition 2.3. The variety $N(W)$ of 2-nilpotent elements is given by $N(W)=\left\{x \in W_{2}: x^{[2]}=0\right\}=\bigcup_{i=1}^{3} N_{W}^{i}$, where

$N_{W}^{1}=\left\{a \eta+b y_{2}+c y_{4}+d y_{5}: a \in k^{*}, b, c, d \in k\right\}$

$N_{W}^{2}=\left\{a \kappa^{[2]}+\frac{b^{2}}{a} \eta+c y_{0}+b y_{1}+d y_{2}+\frac{c^{2}}{a} y_{4}+\frac{b c}{a} y_{5}: a \in k^{*}, b, c, d \in k\right\}$

$N_{W}^{3}=\left\{a y_{2}+b y_{4}+c y_{5}: a, b, c \in k\right\} \subseteq W$.

Moreover,

i) $N_{W}^{1}=\left\{a \eta+y_{2}+c y_{4}+d y_{5}: 0 \neq a, d, c \in k\right\}^{G_{1}} \cup\left\{a \eta+y_{4}+d y_{5}: 0 \neq\right.$ $a, d \in k,\}^{G_{1}} \cup\left\{\eta+d y_{5}: d \in k / \mathbf{Z}_{3}\right\}^{G_{1}}$, here $k / \mathbf{Z}_{3}$ is the set of orbits of the following $\mathbf{Z}_{3}$-action on $k: x \rightarrow \delta x, \delta^{3}=1$.

ii) $N_{W}^{2}=\left\{\kappa^{[2]}\right\}^{G_{1}}$ forms one orbit under the $G_{1}$-action.

iii) $N_{W}^{3}=\left\{y_{2}+b y_{4}+c y_{5}: b, c \in k\right\}^{G_{1}} \cup\left\{y_{4}+c y_{5}: c \in k\right\}^{G_{1}} \cup y_{5}^{G_{1}}$.

We note also that the $G_{1}$-stabilizers of the elements in $N_{W}^{3}$ have dimension 4, but they may be defined over different fields.

Proof. The set $N(W)$ we can describe as the set $I(W)$ but more easy. Consider the set of $G_{1}$-orbits of the natural $G_{1}$-action on $N(W)$. It is easy to see that $\left(N_{W}^{1}\right)^{G_{1}}=N_{W}^{1}$. Let $n=a \eta+b y_{2}+c y_{4}+d y_{5} \in N_{W}^{1}$ 
and $b \neq 0$. Then we can find a diagonal automorphism $\phi=\phi(\alpha, 0,0,0,0)$ such that $n^{\phi}=a_{1} \eta+y_{2}+c_{1} y_{4}+d_{1} y_{5}$. Note that for all $\alpha_{1}, \alpha_{2}, \alpha_{3}, \alpha_{4} \in k$ we have $n^{\phi}=n^{\phi\left(\alpha, \alpha_{1}, \alpha_{2}, \alpha_{3}, \alpha_{4}\right)}$. If $n^{\phi}=\left(a_{2} \eta+y_{2}+c_{2} y_{4}+d_{2} y_{5}\right)^{\phi(\beta, 0,0,0,0)}$, then $\beta^{2}=1$ and $\phi(\beta, 0,0,0,0)=1$. It means that $a_{1} \eta+y_{2}+c_{1} y_{4}+d_{1} y_{5}$ is the unique representative of its $G_{1}$-orbit.

Analogously we proceed in the case $b=0, c \neq 0$. Suppose that $b=c=0$. As above we can find a diagonal automorphism $\phi$ such that $\left(a \eta+d y_{5}\right)^{\phi}=$ $\eta+d_{1} y_{5}$. Let $\psi=\phi(\beta, 0,0,0,0)$ and $\left(\eta+d_{1} y_{5}\right)^{\phi}=\eta+d_{2} y_{5}$. Therefore, $\beta^{6}=1$ and $\beta^{-5} d_{1}=d_{2}$. Then $\beta=\delta \in k, \delta^{3}=1, \beta^{-5}=\delta$, and $d_{1}, d_{2}$ are contained in the same $\mathbf{Z}_{3}$-orbit.

The other cases may be considered analogously.

\section{The Kostrikin-Dzhumadil'daev algebras}

The Kostrikin-Dzhumadil'daev Lie algebras $L(\varepsilon)$ (or $K D$-algebras, for brevity) of dimension 7 form a family depending on one parameter $\varepsilon \in$ $k$ (see Example 7.2 of $[\mathrm{K}]$ ). The multiplication table of basis elements in $L(\varepsilon)$ is as follows:

A $K D$-algebra $L(\varepsilon)$

\begin{tabular}{|c||c|c|c|c|c|c|c|}
\hline \hline & \multicolumn{2}{|c||}{$L(\varepsilon)_{-1}$} & \multicolumn{2}{c|}{$L(\varepsilon)_{0}$} & \multicolumn{2}{|c|}{$L(\varepsilon)_{1}$} & $L(\varepsilon)_{2}$ \\
\hline \hline & $u_{0}$ & $u_{1}$ & $e_{0}$ & $e_{1}$ & $f_{0}$ & $f_{1}$ & $g$ \\
\hline$u_{0}$ & $\cdot$ & 0 & $\varepsilon u_{0}$ & $\bar{\varepsilon} u_{1}$ & $e_{0}$ & $e_{1}$ & $f_{1}$ \\
\hline$u_{1}$ & 0 & $\cdot$ & $\bar{\varepsilon} u_{1}$ & $\varepsilon u_{0}$ & $e_{1}$ & $e_{0}$ & $f_{0}$ \\
\hline$e_{0}$ & $\varepsilon u_{0}$ & $\bar{\varepsilon} u_{1}$ & $\cdot$ & $e_{1}$ & $\varepsilon f_{0}$ & $\bar{\varepsilon} f_{1}$ & $g$ \\
\hline$e_{1}$ & $\bar{\varepsilon} u_{1}$ & $\varepsilon u_{0}$ & $e_{1}$ & $\cdot$ & $\varepsilon f_{1}$ & $\bar{\varepsilon} f_{0}$ & 0 \\
\hline$f_{0}$ & $e_{0}$ & $e_{1}$ & $\varepsilon f_{0}$ & $\varepsilon f_{1}$ & $\cdot$ & $g$ & 0 \\
\hline$f_{1}$ & $e_{1}$ & $e_{0}$ & $\bar{\varepsilon} f_{1}$ & $\bar{\varepsilon} f_{0}$ & $g$ & $\cdot$ & 0 \\
\hline$g$ & $f_{1}$ & $f_{0}$ & $g$ & 0 & 0 & 0 & $\cdot$ \\
\hline \hline
\end{tabular}

Firstly note that for $\varepsilon=0$ or $\varepsilon=1$ the algebra $L(\varepsilon)$ is semi-simple but not simple. It is an easy exercise to prove that $L_{0}$ and $L_{1}$ are isomorphic. For $\varepsilon \notin\{0,1\}$, the following theorem holds.

Theorem 3.1. Given $\varepsilon \notin\{0,1\}$, the corresponding simple KD-algebra $L(\varepsilon)$ is isomorphic to the Hamiltonian algebra $H_{2}=H((2,1), \omega)$.

Proof. For $\varepsilon \in k \backslash\{0,1\}$, consider the Lie algebra $L(\varepsilon)$ as given above and apply the following changing of basis: $V_{0}=\sqrt{\varepsilon \bar{\varepsilon}}\left(u_{0}+u_{1}\right), V_{1}=$ $\varepsilon u_{0}+\bar{\varepsilon} u_{1}, \quad F_{0}=f_{0}+f_{1}, \quad F_{0}=\frac{1}{\sqrt{\varepsilon \bar{\varepsilon}}}\left(\bar{\varepsilon} f_{0}+\varepsilon f_{1}\right), \quad E_{1}=\frac{e_{1}}{\sqrt{\varepsilon \bar{\varepsilon}}}, E_{0}=$ 
$e_{0}+e_{1}, G=\frac{g}{\sqrt{\varepsilon \bar{\varepsilon}}}$. Hence, $L(\varepsilon)$ is isomorphic to the Lie algebra $K=$ $\left\langle V_{0}, V_{1}, E_{0}, E_{1}, F_{0}, F_{1}, G\right\rangle$ given by the Lie multiplication table below. It is easy to see that a basis of the 2-closure $K_{2}$ may be chosen as follows: $\left\{t, m, n, V_{0}, V_{1}, E_{0}, E_{1}, F_{0}, F_{1}, G\right\}$ and the multiplication table in $K_{2}$ is the following:

The 2-closure $K_{2}$ of the $K D$-algebra $K$

\begin{tabular}{|c||c|c|c|c|c|c|c|c|c|c|}
\hline \hline & $t$ & $m$ & $n$ & $V_{0}$ & $V_{1}$ & $E_{1}$ & $E_{0}$ & $F_{1}$ & $F_{0}$ & $G$ \\
\hline \hline$t$ & $t$ & 0 & 0 & $V_{0}$ & $V_{1}$ & 0 & 0 & $F_{1}$ & $F_{0}$ & 0 \\
\hline$m$ & 0 & 0 & $E_{0}$ & 0 & 0 & 0 & 0 & $V_{1}$ & $V_{0}$ & $E_{1}$ \\
\hline$n$ & 0 & $E_{0}$ & 0 & 0 & $F_{1}$ & $G$ & 0 & 0 & 0 & 0 \\
\hline$V_{0}$ & $V_{0}$ & 0 & 0 & 0 & 0 & $V_{1}$ & 0 & 0 & $E_{0}$ & $F_{1}$ \\
\hline$V_{1}$ & $V_{1}$ & 0 & $F_{1}$ & 0 & $m$ & $V_{0}$ & $V_{1}$ & $E_{0}$ & $E_{1}$ & $F_{0}$ \\
\hline$E_{1}$ & 0 & 0 & $G$ & $V_{1}$ & $V_{0}$ & $t$ & $E_{1}$ & $F_{0}$ & $F_{1}$ & 0 \\
\hline$E_{0}$ & 0 & 0 & 0 & 0 & $V_{1}$ & $E_{1}$ & $E_{0}$ & $F_{1}$ & 0 & $G$ \\
\hline$F_{1}$ & $F_{1}$ & $V_{1}$ & 0 & 0 & $E_{0}$ & $F_{0}$ & $F_{1}$ & $n$ & $G$ & 0 \\
\hline$F_{0}$ & $F_{0}$ & $V_{0}$ & 0 & $E_{0}$ & $E_{1}$ & $F_{1}$ & 0 & $G$ & $n$ & 0 \\
\hline$G$ & 0 & $E_{1}$ & 0 & $F_{1}$ & $F_{0}$ & 0 & $G$ & 0 & 0 & 0 \\
\hline \hline
\end{tabular}

Note that $K$ has a Cartan subalgebra $C=k\left\{E_{0}, F_{0}, V_{0}\right\}$ of toral rank one (but the absolute toral rank of $C$ is equal to two!) Recall that Skryabin's Theorem 6.2 [Sk] asserts (in particular) that every finite dimensional simple Lie algebra $L$ over a field of characteristic 2 with a Cartan subalgebra $C$ of toral rank one is isomorphic to a Hamiltonian algebra if $\operatorname{dimL} / L_{0}=$ 2 , where $L_{0}$ is a maximal subalgebra that contains $C$. In our case $K_{0}=$ $\operatorname{Span}_{k}\left\{E_{0}, F_{0}, V_{0}, G, F_{1}\right\}$ and $\operatorname{dim} K / K_{0}=2$. Hence $K$ is a Hamiltonian algebra by Skryabin's Theorem. On the other hand there exists a unique 7-dimensional Hamiltonian algebra $H_{2}=H((2,1), \omega)$, where $\omega=(1+$ $\left.x_{1}^{(3)} x_{2}\right) d x_{1} \wedge d x_{2}$ is a non-standard 2-form.

From now on we will denote a $K D$-algebra $L(\varepsilon)$, for $\varepsilon \notin\{0,1\}$, simply by $K$ and its 2 -closure by $K_{2}$, as in the theorem above.

3.1. The group of 2-automorphisms $G_{2}=A u t_{k, 2}\left(K_{2}\right)$. 
Proposition 3.1. The group of 2-automorphisms $G_{2}$ of the Lie 2-algebra $K_{2}$ is defined on its basis elements, for $\varphi=\varphi(a, b, c) \in G_{2}$ and $a \neq 0$, by:

$$
\begin{aligned}
\varphi: E_{0} & \longmapsto E_{0}+a^{-2} b^{2} G \\
G & \longmapsto a^{2} G \\
F_{0} & \longmapsto a F_{0} \\
F_{1} & \longmapsto a F_{1}+b G \\
E_{1} & \longmapsto E_{1}+a^{-1} b F_{1}+c G \\
V_{0} & \longmapsto a^{-1} V_{0}+a^{-2} b E_{0}+a^{-3} b^{2} F_{1}+a^{-3} b^{2} F_{0}+a^{-4} b^{3} G \\
V_{1} & \longmapsto a^{-1} V_{1}+a^{-2} b E_{1}+a^{-1} c F_{1}+a^{-3} b^{2} F_{0}+\left(a^{-2} b c+\right. \\
& a^{-4} b^{3} G \\
n & \longmapsto a^{2} n \\
t & \longmapsto t+a^{-2} b^{2} n+a^{-1} b F_{0} \\
m & \longmapsto a^{-2} m+a^{-4} b^{2} t+\left(a^{-2} c^{2}+a^{-6} b^{4}\right) n+a^{-3} b V_{0}+ \\
& a^{-4} b^{2} E_{1}+a^{-2} c E_{0}+a^{-5} b^{3} F_{1}+a^{-5} b^{3} F_{0}+ \\
& a^{-4} b^{2} c G .
\end{aligned}
$$

Note that $\operatorname{dim}_{k} G_{2}=3$ for every field $k$ of characteristic 2 .

Proof. Let $\phi$ be an automorphism of $K_{2}$. Then $\left\{x \in K: x^{[2]}=x\right\}^{\phi}=$ $\left\{x \in K: x^{[2]}=x\right\}=\left\{E_{0}+a G: a \in k\right\}$; in particular, $E_{0}^{\phi}=E_{0}+a G$.

For all $a_{1}, a_{2} \in k$, the map $E_{0}+a_{2} G \rightarrow E_{0}+a_{1} G$ may be extended to an automorphism $\psi=\psi_{a_{1}, a_{2}}$. Hence, $E_{0}^{\phi \psi_{0, a}}=E_{0}$ and we may assume that $E_{0}^{\phi}=E_{0}$. Let $S=A n n_{K} E_{0}=\operatorname{Span}_{k}\left\{V_{0}, E_{0}, F_{0}\right\}$. Then $S^{\phi}=S$ and $V_{0}^{\phi}=a V_{0}, 0 \neq a \in k$, since $k V_{0}=\left\{x \in S: x^{[2]}=0\right\}$. It is easy to see that the map $\tau: E_{0} \rightarrow E_{0}, V_{0} \rightarrow a^{-1} V_{0}, V_{1} \rightarrow a^{-1} V_{1}, F_{1} \rightarrow a F_{1}, F_{0} \rightarrow a F_{0}$, $G \rightarrow a^{2} G$ is an automorphism. Therefore, $V_{0}^{\phi \tau}=V_{0}$ and we may suppose that $E_{0}^{\phi}=E_{0}, V_{0}^{\phi}=V_{0}$. Since $\left\{x \in S: x^{[4]}=0\right\}^{\phi}=\left\{x \in S: x^{[4]}=0\right\}=$ $k V_{0} \cup k F_{0}$, we have $F_{0}^{\phi}=F_{0}$. Analogously, if $T=\left\{x \in K:\left[x, E_{0}\right]=x\right\}$ then $A n n_{T} F_{0}=k G$ and $G^{\phi}=G$. We have $E_{1}^{\phi}=E_{1}+a F_{1}+b G$, then

$$
\left[E_{1}^{\phi}, F_{0}^{\phi}\right]=\left[E_{1}, F_{0}\right]^{\phi}=F_{1}^{\phi}=F_{1}=\left[E_{1}+a F_{1}+b G, F_{0}\right]=F_{1}+a G,
$$

and $a=0$. Furthermore,

$$
V_{1}^{\phi}=\left[E_{1}, V_{0}\right]^{\phi}=\left[E_{1}^{\phi}, V_{0}^{\phi}\right]=\left[E_{1}+b G, V_{0}\right]=V_{1}+b F_{1} .
$$

It is easy to see that $\phi$ is an automorphism. Hence, $\operatorname{dim} G_{2}=3$.

\subsection{Idempotent and Nilpotent Elements of $K_{2}$.}

Proposition 3.2. For the 2-closure $K_{2}$ of the $K D$-algebra, the variety of idempotent elements $I(K)=\left\{x \in k_{2}: 0 \neq x^{[2]}=x\right\}$ is given by 


$$
\begin{aligned}
& I_{K}^{1}=\bigcup_{i=1}^{6} I_{K}^{i}, \text { where } \\
& I_{K}^{1}=\left\{\alpha^{2} t+\xi^{-2} m+\xi^{2}(b+\bar{\alpha} \bar{a})^{2} n+a \xi^{-1} V_{0}+\xi^{-1} V_{1}+\alpha \bar{\alpha} E_{1}+b E_{0}+\right. \\
& \xi(b+\bar{\alpha}(\alpha a+\bar{\alpha})) F_{1}+\xi \bar{\alpha}(\alpha a+a+\alpha) F_{0}+\xi^{2} \bar{\alpha}(b \alpha+\alpha a+a) G: \alpha, a, b \in \\
& \left.k, \xi \in k^{*}\right\} \\
& I_{K}^{2}=\left\{t+\xi^{2}\left(b^{2}+b+c\right)^{2} n+\xi^{-1} V_{0}+b E_{0}+c \xi F_{1}+\xi\left(b^{2}+b\right) F_{0}+\xi^{2} b c G:\right. \\
& \xi, b, c \in k\} \\
& I_{K}^{3}=\left\{t+\xi^{-1} c^{2} n+E_{0}+c \xi F_{0}+\xi^{2} d G: \xi, c, d \in k\right\} \\
& I_{K}^{4}=\left\{t+\xi^{2}\left(c_{0}+c_{1}\right)^{2} n+\xi c_{1} F_{1}+c_{0} \xi F_{0}+\xi^{2} c_{0} c_{1} G: \xi, c_{0}, c_{1} \in k\right\} \\
& I_{K}^{5}=\left\{\delta t+\delta a^{2} n+a\left(\delta F_{0}+F_{1}\right)+E_{1}+E_{0}+d G: \delta^{2}+\delta+1=0, a, d \in k\right\} \\
& I_{K}^{6}=\left\{E_{0}+d G: d \in k\right\} .
\end{aligned}
$$

Proposition 3.3. The variety of nilpotent elements $N(K)=\{x \in$ $\left.K_{2}: x^{[2]}=0\right\}$ is described as follows: $N(K)=\bigcup_{i=1}^{6} N_{K}^{i}$, where

$N_{K}^{1}=\left\{t+\beta m+\left(c^{2}+\beta d^{2}\right) n+\beta c V_{0}+E_{1}+\beta d E_{0}+c\left(F_{1}+F_{0}\right)+d G\right.$ : $\beta, d, c \in k\}$

$N_{K}^{2}=\left\{t+c^{2} n+E_{1}+c\left(F_{0}+F_{1}\right)+d G: d, c \in k\right\}$

$N_{K}^{3}=\{n+d G: d \in k\}, \quad N_{K}^{4}=\left\{n+a V_{0}: a \in k\right\}$

$N_{K}^{5}=\left\{n+b^{3} V_{0}+d b^{2} E_{0}+b d^{2}\left(F_{0}+F_{1}\right)+d^{3} G: d, b \in k\right\}$

$N_{K}^{6}=\left\{\alpha^{3} V_{0}+\alpha^{2} \gamma E_{0}+\alpha \gamma^{2}\left(F_{0}+F_{1}\right)+\gamma^{3} G: \alpha, \gamma \in k\right\}$.

Proofs of Propositions 3.2 and 3.3 are analogous to the proof of Proposition 2.2.

Proposition 3.4. The $G_{2}$-orbits of the variety $I(K)=\bigcup_{i=1}^{7} O I_{K}^{i}$ are $I_{K}^{1}=O I_{K}^{1}=\cup_{\lambda \in k} O I_{K, \lambda}^{1}, O I_{K, \lambda}^{1}=\left\{t+m+\lambda V_{0}+V_{1}\right\}^{G_{2}}$

$I_{K}^{2}=O I_{K}^{2}=\cup_{b \in k} O I_{K, b}^{2}, O I_{K, b}^{2}=\left\{t+V_{0}+b E_{0}+b \bar{b}\left(F_{1}+F_{0}\right)+\right.$ $\left.b^{2} \bar{b} G\right\}^{G_{2}}$

$$
\begin{aligned}
& I_{K}^{3}=O I_{K}^{3}=\cup_{d \in k} O I_{K, d}^{3}, O I_{K, d}^{3}=\left\{t+E_{0}+d G\right\}^{G_{2}} \\
& I_{K}^{4}=O I_{K}^{4} \cup O I_{K}^{5}, O I_{K}^{4}=\{t\}^{G_{2}} O I_{K}^{5}=\left\{t+F_{1}+F_{0}+G\right\}^{G_{2}} \\
& I_{K}^{5}=O I_{K}^{6}=\left\{\delta t+E_{1}+E_{0}: \delta^{2}+\delta+1=0\right\}^{G_{2}} \\
& I_{K}^{6}=O I_{K}^{7}=\left\{E_{0}\right\}^{G_{2}} .
\end{aligned}
$$

Proof. Show that $I_{K}^{1}=\cup_{\lambda \in k} O I_{K, \lambda}^{1}$. Denote by $\phi(a, b, c)$ an automorphism from Proposition 3.1. Let $a_{1}=\xi, b_{1}=\xi^{2}(1+\alpha), c_{1}=\xi\left(\xi^{-3} b^{2}+\right.$ $\xi(b+\bar{a} \bar{\alpha})), \lambda=a_{1}\left(a \xi^{-1}+a_{1}^{-3} b_{1}\right)$. Then by direct calculation we get

$$
\begin{aligned}
& \left(t+m+\lambda V_{0}+V_{1}\right)^{\phi\left(a_{1}, b_{1}, c_{1}\right)}=\alpha^{2} t+\xi^{-2} m+\xi^{2}(b+\bar{\alpha} \bar{a})^{2} n+a \xi^{-1} V_{0}+ \\
& \xi^{-1} V_{1}+\alpha \bar{\alpha} E_{1}+b E_{0}+\xi(b+\bar{\alpha}(\alpha a+\bar{\alpha})) F_{1}+\xi \bar{\alpha}(\alpha a+a+\alpha) F_{0}+\xi^{2} \bar{\alpha}(b \alpha+
\end{aligned}
$$


$\alpha a+a) G \in I_{K, \lambda}^{1}$.

The other cases may be considered analogously. For example,

$I_{K}^{6}=\left\{\delta t+E_{1}+E_{0}: \delta^{2}+\delta+1=0,\right\}^{G_{2}}$,
since $\left(\delta t+E_{1}+E_{0}\right)^{\phi\left(1, a, d+a^{2}\right)}=\delta t+\delta a^{2} n+a\left(\delta F_{0}+F_{1}\right)+E_{1}+E_{0}+d G$.

Note that $N_{K}^{5} \subset K$. We have the following result on the varieties of nilpotent and idempotent elements.

Theorem 3.2. The varieties $I(A)$ and $N(A)$, for $A \in\{W, K\}$, are irreducible.

Proof. We write a detailed proof for the variety $I(K)$ and leave the other cases to the reader. It suffices to prove that the first orbit includes in its closure (in the Zariski topology) all the other orbits. Observe that a generic element of the orbit $\operatorname{orb}(1)$, in projective coordinates, is written as: $f(\lambda, \xi, \alpha, b, a)=\lambda^{4} \xi^{2} \alpha^{2} t+\lambda^{8} m+\xi^{4}\left(b^{2} \lambda^{2}+(\lambda+\alpha)^{2}(\lambda+a)^{2}\right) n+\lambda^{6} a \xi V_{0}+$ $\lambda^{7} \xi V_{1}+\lambda^{4} \xi^{2} \alpha(\lambda+\alpha) E_{1}+\lambda^{5} \xi^{2} b E_{0}+\lambda^{2} \xi^{3}\left(b \lambda^{2}+(\lambda+\alpha)\left(\alpha a+\lambda^{2}+\alpha \lambda\right)\right) F_{1}+$ $\lambda^{2} \xi^{3}(\lambda+\alpha)(\lambda \alpha+a \alpha+a \lambda) F_{0}+\lambda \xi^{4}(\lambda+\alpha)(b \alpha+a \alpha+a \lambda) G$.

1) Now we make the following substitutions: $b=\frac{1}{\lambda}, \xi=\frac{\lambda^{3}}{(1+\lambda)^{3}}, a=$ $\frac{1}{\lambda(\lambda+1)}, \alpha=1$ and $\bar{\lambda}=\lambda+1$. Hence,

$$
\begin{aligned}
f\left(\lambda, \frac{\lambda^{3}}{\bar{\lambda}^{3}}, 1, \lambda^{-1}, \frac{1}{\lambda \bar{\lambda}}\right)= & \frac{\lambda^{10}}{\bar{\lambda}^{6}}\left(t+n+E_{0}+F_{0}\right)+\lambda^{8} m+\frac{\lambda^{8}}{\bar{\lambda}^{4}} V_{0}+\frac{\lambda^{10}}{\bar{\lambda}^{3}} V_{1}+ \\
& \frac{\lambda^{10}}{\bar{\lambda}^{5}} E_{1}+\frac{\lambda^{10}}{\bar{\lambda}^{6}} F_{1} .
\end{aligned}
$$

Let $\chi$ be the closure (in the Zariski topology) of the orbit $O I_{K}^{1}$. Then we have $\lambda^{10}\left(t+n+E_{0}+F_{0}\right)+\bar{\lambda}\left(\bar{\lambda}^{5} \lambda^{8} m+\bar{\lambda} \lambda^{8} V_{0}+\bar{\lambda}^{2} \lambda^{10} V_{1}+\bar{\lambda} \lambda^{10} E_{1}\right)+$ $\lambda^{10} F_{1} \in \chi$. Hence, for $\lambda=1$, one gets $u=t+n+E_{0}+F_{0} \in \chi$. Applying the automorphism $\varphi(a, b, c)$ with $a^{2}=b, c=0$ to $u$ we obtain $u^{\varphi}=t+E_{0}+a^{2} G \in \chi$. Therefore, $O I_{K}^{3}$ is contained in $\chi$.

2) Putting $\xi=a, \lambda=\alpha, \alpha_{1}=\frac{\alpha}{a}, b_{1}=\frac{b}{\alpha}$, we have

$$
\begin{aligned}
f=f(\alpha, a, \alpha, b, a)= & \alpha^{6} a^{2} t+\alpha^{8} m+\alpha^{2} b^{2} a^{4} n+\alpha^{6} a^{2} V_{0}+\alpha^{7} a V_{1}+ \\
& \alpha^{5} a^{2} b E_{0}+\alpha^{4} a^{3} b F_{1} .
\end{aligned}
$$

Hence, $\frac{f}{\alpha^{6} a^{2}}=\left(t+V_{0}\right)+\alpha_{1}^{2} m+\alpha_{1} V_{1}+\left(\frac{b_{1}}{\alpha_{1}}\right)^{2} n+b_{1} E_{0}+\frac{b_{1}}{\alpha_{1}} F_{1}=$ $\bar{f}\left(\alpha_{1}, b_{1}\right)$. Therefore, $\bar{f}\left(\alpha_{1}, \tau \alpha_{1}\right)=\left(t+V_{0}+\tau^{2} n+\tau F_{1}\right)+\alpha_{1}^{2} m+$ 
$\alpha_{1} V_{1}+\alpha_{1} E_{0}$. Thus, for $\alpha=0$, one gets $g=t+V_{0}+\tau^{2} n+\tau F_{1} \in \chi$. Applying the automorphism $\varphi=\varphi(1, \tau, 0)$ to $g$, we obtain $g^{\varphi}=t+V_{0}+$ $\tau E_{0}+\tau \bar{\tau}\left(F_{0}+F_{1}\right)+\tau^{2} \bar{\tau} G \in \chi$. Therefore, $O I_{K}^{2}$ is also contained in $\chi$.

3) Now put $b=0$ and $\lambda=a$ in $f$. Then

$f=a^{4} \xi^{2} \alpha^{2} t+a^{8} m+a^{7} \xi\left(V_{0}+V_{1}\right)+a^{4} \xi^{2} \alpha(a+\alpha) E_{1}+a^{4} \xi^{3}(a+\alpha)^{2}\left(F_{0}+\right.$ $\left.F_{1}\right)+a^{2} \xi^{4}(a+\alpha)^{2} G$.

Substituting $a_{1}=\frac{a}{\xi}, a_{2}=\frac{a}{\alpha}$ we have:

$g=\frac{f}{a^{4} \xi^{2} \alpha^{2}}=t+a_{1}^{2} a_{2}^{2} m+\left(1+a_{2}\right) E_{1}+a_{1} a_{2}^{2}\left(V_{0}+V_{1}\right)+\frac{\left(1+a_{2}\right) a_{2}}{a_{1}}\left(F_{0}+\right.$ $\left.F_{1}\right)+\frac{\left(1+a_{2}\right)^{2}}{a_{1}^{2}} G$.

For $a_{1}=a_{2}+1$ one gets $g=t+{\overline{a_{2}}}^{2} a_{2}^{2} m+\overline{a_{2}} E_{1}+\overline{a_{2}} a_{2}^{2}\left(V_{0}+V_{1}\right)+$ $a_{2}\left(F_{0}+F_{1}\right)+G$. Hence, if $a_{2}=1$, then $g=t+F_{0}+F_{1}+G \in \chi$, that is, $O I_{K}^{4}$ is contained in $\chi$.

4) Let $\lambda=\tau \alpha=b, a=\tau^{2} \alpha$ and so, as $\tau^{2}+\tau=1$, we have $\alpha+\lambda=\tau^{2} \alpha$. Hence,

$$
f\left(\tau \alpha, \xi, \tau \alpha, \tau^{2} \alpha\right)=\tau \alpha^{6} \xi^{2} t+\alpha^{6} \xi^{2}\left(E_{0}+E_{1}\right)+\tau^{2} \alpha^{8} m+\tau^{2} \alpha^{7} \xi V_{0}+
$$
$\alpha^{7} \xi V_{1}$.

By substituting $\rho=\frac{\alpha}{\xi}$, one gets $\frac{f}{\alpha^{6} \xi^{2}}=\left(\tau t+E_{0}+E_{1}\right)+\tau^{2} \rho^{2} m+$ $\tau^{2} \rho V_{0}+\rho V_{1}$. For $\rho=0$ we have $\tau t+E_{0}+E_{1} \in \chi$. Therefore, $O I_{K}^{6} \subset \chi$.

5) Applying the automorphism $\varphi=\varphi(a, 0,0)$ to $g=t+F_{0}+F_{1}+G$, we get $g^{\varphi}=t+a\left(F_{0}+F_{1}\right)+a^{2} G$. Hence, for $a=0$, the orbit of $t$ is also contained in $\chi$.

6) Finally, to prove that $O I_{K}^{7} \subset \chi$, consider $\frac{1}{b}\left(t+V_{0}+b E_{0}+b \bar{b}\left(F_{1}+\right.\right.$ $\left.\left.F_{0}\right)+b^{2} \bar{b} G\right)=a t+a V_{0}+E_{0}+\bar{b}\left(F_{1}+F_{0}\right)+b \bar{b} G$, with $a \in k$. In this way, for $a=0, b=1$, in the Zariski topology, $E_{0}$ lies in the closure of $O I_{K}^{2}$, which is contained in $\chi$.

3.3. Cartan decompositions. An interesting and important problem for a Lie 2-algebra is the classification of its Cartan subalgebras up to automorphisms. Here we give some examples of Cartan subalgebras of $K_{2}$ and $W_{2}$ such that the corresponding Cartan decomposition is defined over a field $\mathbf{F}_{4}$ for $W_{2}$ and over $\mathbf{F}_{2}$ for the algebra $K_{2}$.

Conjecture 3.1. A toral subalgebra of $A_{2}$ of dimension 3 always has an idempotent from $I_{A}^{1}, A \in\{W, K\}$. Let $T$ be a toral subalgebra of $W_{2}$ of dimension 3. Suppose that $T$ is defined over a field $\mathbf{F}$, then $\mathbf{F}_{4} \subseteq \mathbf{F}$. 
A particular example of a toral Cartan subalgebra $T$ of $W_{2}$ is generated by $\left\{t_{1}, t_{2}, t_{3}\right\}$ where $t_{1}=\eta+y_{0}+y_{3}, t_{2}=\kappa^{[2]}+\kappa+y_{-1}+y_{1}+y_{2}, t_{3}=$ $\delta^{2}\left(\kappa+y_{1}\right)+\delta\left(\kappa^{[2]}+y_{-1}+y_{2}\right)$, with $\delta^{2}+\delta+1=0, \delta^{3}=1, \delta \in k^{*}$.

Let $\mathcal{G}=\langle\alpha, \beta, \gamma\rangle$ be an elementary abelian group of order 8. A Cartan decomposition of $W_{2}$ with respect to $T$ is given by

$$
W_{2}=T \oplus \sum_{\xi \in \mathcal{G}} \oplus L_{\xi}
$$

where $L_{\xi}=\left\langle e_{\xi}\right\rangle$ and $e_{\alpha}=y_{-1}+y_{2}, e_{\beta}=\delta^{2}\left(y_{0}+y_{3}\right)+\left(y_{2}+y_{5}\right)+\delta y_{2}$, $e_{\gamma}=y_{0}+y_{2}+y_{3}+y_{4}+y_{5}, e_{\alpha+\beta}=y_{-1}+y_{2}+y_{5}+\delta\left(y_{1}+y_{4}\right)+\delta^{2} y_{3}$, $e_{\alpha+\gamma}=y_{-1}+y_{1}+y_{2}+y_{3}+y_{4}+y_{5}, e_{\beta+\gamma}=\delta\left(y_{0}+y_{3}\right)+\left(y_{2}+y_{5}\right)+\delta^{2} y_{4}$ and $e_{\alpha+\beta+\gamma}=y_{-1}+y_{2}+y_{5}+\delta y_{3}+\delta^{2}\left(y_{1}+y_{4}\right)$.

In the diagonal of the table below, we present the elements $e_{\xi}^{[2]}, \xi \in$ $\mathcal{G}$ and $\tilde{t}=t_{3}+\delta\left(t_{1}+t_{2}\right), \check{t}=\delta^{2} t_{1}+\delta t_{2}+t_{3}$. Note that this Cartan decomposition occurs over a field $k$ with four elements.

\begin{tabular}{|c||c|c|c|c|c|c|c|}
\hline \hline & $e_{\alpha}$ & $e_{\beta}$ & $e_{\gamma}$ & $e_{\alpha+\beta}$ & $e_{\alpha+\gamma}$ & $e_{\beta+\gamma}$ & $e_{\alpha+\beta+\gamma}$ \\
\hline \hline$e_{\alpha}$ & $t_{3}+\delta t_{2}$ & $\delta^{2} e_{\alpha+\beta}$ & $e_{\alpha+\gamma}$ & $\delta^{2} e_{\beta}$ & $e_{\gamma}$ & $\delta e_{\alpha+\beta+\gamma}$ & $\delta e_{\beta+\gamma}$ \\
\hline$e_{\beta}$ & $\delta^{2} e_{\alpha+\beta}$ & $\delta t_{1}$ & 0 & $\delta^{2} e_{\alpha}$ & $\delta^{2} e_{\alpha+\beta+\gamma}$ & 0 & $e_{\alpha+\gamma}$ \\
\hline$e_{\gamma}$ & $e_{\alpha+\gamma}$ & 0 & $t_{1}$ & $e_{\alpha+\beta+\gamma}$ & $e_{\alpha}$ & 0 & $e_{\alpha+\beta}$ \\
\hline$e_{\alpha+\beta}$ & $\delta^{2} e_{\beta}$ & $\delta^{2} e_{\alpha}$ & $e_{\alpha+\beta+\gamma}$ & $\tilde{t}$ & $\delta e_{\beta+\gamma}$ & $\delta e_{\alpha+\gamma}$ & $e_{\gamma}$ \\
\hline$e_{\alpha+\gamma}$ & $e_{\gamma}$ & $\delta^{2} e_{\alpha+\beta+\gamma}$ & $e_{\alpha}$ & $\delta e_{\beta+\gamma}$ & $t_{3}+t_{1}+\delta t_{2}$ & $\delta e_{\alpha+\beta}$ & $\delta^{2} e_{\beta}$ \\
\hline$e_{\beta+\gamma}$ & $\delta e_{\alpha+\beta+\gamma}$ & 0 & 0 & $\delta e_{\alpha+\gamma}$ & $\delta e_{\alpha+\beta}$ & $\delta^{2} t_{1}$ & $\delta e_{\alpha}$ \\
\hline$e_{\alpha+\beta+\gamma}$ & $\delta e_{\beta+\gamma}$ & $\delta^{2} e_{\alpha+\gamma}$ & $e_{\alpha+\beta}$ & $e_{\gamma}$ & $\delta^{2} e_{\beta}$ & $\delta e_{\alpha}$ & $\tilde{t}$ \\
\hline \hline
\end{tabular}

Consider the following elements of $K_{2}$ :

$$
\begin{array}{lll}
t_{1}=m+E_{0}+V_{1} & a_{1}=E_{1}+F_{0}+G & b_{1}=V_{0}+F_{0}+G \\
t_{2}=t+n+F_{1} & a_{2}=E_{0}+V_{1} & b_{2}=E_{0}+F_{1} \\
t_{3}=t+m+V_{1} & a_{3}=E_{1}+F_{0} & b_{3}=V_{0} \\
& & b=V_{1}+E_{1}+F_{1}
\end{array}
$$

Let $T=\left\langle t_{i}: i=1,2,3\right\rangle$ with $t_{i}^{[2]}=t_{i}$. It is easy to verify that $\left[a_{i}, t_{j}\right]=$ $\delta_{i j} a_{i}, I(K)=\left\{t \in K: t^{[2]}=t\right\}=\left\{\alpha a_{1}+a_{2}+\alpha a_{3}+b_{2}+b: \alpha \in k\right\}$. This gives a decomposition of $K_{2}$ on root spaces, and we have the following Lie multiplication table, where in the diagonal are written the elements $x^{[2]}$. Observe that this multiplication is defined over the prime field $\mathbb{F}_{2}$. 


\begin{tabular}{|c||c|c|c|c|c|c|c|c|c|c|}
\hline \hline & $t_{1}$ & $t_{2}$ & $t_{3}$ & $a_{1}$ & $a_{2}$ & $b_{1}$ & $a_{3}$ & $b_{2}$ & $b_{3}$ & $b$ \\
\hline \hline$t_{1}$ & $t_{1}$ & 0 & 0 & $a_{1}$ & 0 & $b_{1}$ & 0 & $b_{2}$ & 0 & $b$ \\
\hline$t_{2}$ & 0 & $t_{2}$ & 0 & 0 & $a_{2}$ & $b_{1}$ & 0 & 0 & $b_{3}$ & $b$ \\
\hline$t_{3}$ & 0 & 0 & $t_{3}$ & 0 & 0 & 0 & $a_{3}$ & $b_{2}$ & $b_{3}$ & $b$ \\
\hline$a_{1}$ & $a_{1}$ & 0 & 0 & $t_{2}$ & $b_{1}$ & $a_{2}$ & 0 & $a_{3}$ & $b$ & $b_{3}$ \\
\hline$a_{2}$ & 0 & $a_{2}$ & 0 & $b_{1}$ & $t_{1}$ & $a_{1}$ & $b_{3}$ & $b$ & 0 & $b_{2}$ \\
\hline$b_{1}$ & $b_{1}$ & $b_{1}$ & 0 & $a_{2}$ & $a_{1}$ & $t_{1}+t_{2}+t_{3}$ & $b$ & 0 & $b_{2}$ & $a_{3}$ \\
\hline$a_{3}$ & 0 & 0 & $a_{3}$ & 0 & $b_{3}$ & $b$ & $t_{2}$ & $a_{1}$ & $a_{2}$ & $b_{1}$ \\
\hline$b_{2}$ & $b_{2}$ & 0 & $b_{2}$ & $a_{3}$ & $b$ & 0 & $a_{1}$ & $t_{1}+t_{2}+t_{3}$ & 0 & $a_{2}$ \\
\hline$b_{3}$ & 0 & $b_{3}$ & $b_{3}$ & $b$ & 0 & $b_{2}$ & $a_{2}$ & 0 & 0 & 0 \\
\hline$b$ & $b$ & $b$ & $b$ & $b_{3}$ & $b_{2}$ & $a_{3}$ & $b_{1}$ & $a_{2}$ & 0 & $t_{2}+t_{3}$ \\
\hline \hline
\end{tabular}

\section{Referências}

[B] BLOCK, R. E., The classification problem for simple Lie algebras of characteristic $p$, in Lie Algebras and Related Topics (LNM Vol. 933), SpringerVerlag, New York, 1982, 38-56.

[BW1] BLOCK, R. E., WiLson, R. L., The restricted simple Lie algebras are of classical or Cartan type, Proc. Nat. Acad. Sci. U.S.A. (1984) 5271-5274.

[BW2] BlOCK, R. E., Wilson, R. L., Classification of restricted simple Lie algebras, J. Algebra 114 (1988), 115-259.

[GP] Grichkov, A.N., Premet, A.A., Simple Lie algebras of absolute toral rank 2 in characteristic 2 (manuscript).

[Ju] Jurman, G., A family of simple Lie algebras in characteristic two, Journal of Algebra 271 (2004) 454-481.

[K] Kostrikin, A. I., The beginnings of modular Lie algebra theory, in: Group Theory, Algebra, and Number Theory (Saarbrücken, 1993), de Gruyter, Berlin, 1996, pp. 13-52.

[PS1] Premet, A. A., Strade, H., Simple Lie algebras of small characteristic: I. Sandwich elements, J . Algebra 189 (1997), 419-480.

[PS2] Premet, A. A., Strade, H., Simple Lie algebras of small characteristic: II. Exceptional roots, J . Algebra 216 (1999), 190-301.

[PS3] PREmet, A. A., STRAde, H., Simple Lie algebras of small characteristic III. The toral rank 2 case, J. Algebra 242 (2001) 236-337.

[R] ReE, R., On generalized Witt algebras, Trans. Amer. Math. Soc. 83 (1956), 510-546.

[Sk] SkRYABIN, S., Toral rank one simple Lie algebras of low characteristics, J . Algebra 200 (1998), 650-700.

[SF] Strade, H., FARnsteiner, R., Modular Lie Algebras and Their Representations, Marcel Dekker, New York, 1988.

[SW] Strade, H., Wilson, R. L., Classification of simple Lie algebras over algebraically closed fields of prime characteristic, Bull. Amer. Math. Soc.24 (1991), 357-362.

[S89] Strade, H., The absolute toral rank of a Lie algebra, Lecture Note in Mathematics, Vol. 1373, Springer-Verlag, Berlin 1989, 1-28.

[S89.1] Strade, H., The classification of the simple modular Lie algebras: I. Determination of the two-sections, Ann. Math. 130 (1989), 643-677. 
[S92] StRADE, H., The classification of the simple modular Lie algebras: II. The toral structure, J. Algebra 151 (1992), 425-475.

[S92.1] Strade, H., The classification of the simple modular Lie algebras: IV. Solving the final case, Trans. Amer. Math. Soc. 350 (1998), 2553-2628.

[W] WANG, Q., On the tori and Cartan subalgebras of Lie algebras of Cartan type, Ph.D. Dissertation, University of Wisconsin, Madison 1992.

[Wi] WiLsOn, R. L. Classification of the restricted simple Lie algebras with toral Cartan subalgebras, J . Algebra 83 (1983), 531-570. 